\title{
Lightning fatalities and injuries in Malaysia from 2008 to 2015
}

\begin{abstract}
This study examined the risk of lightning-related deaths and injuries in Malaysia between January of 2008 to July 2015. The majority of lightning-related injuries and fatalities occurred during the months of April and May. Most lightning related casualties reported in the statistics since 2008 occurred in the states of Melaka and Selangor in the western coastal belt. The majority of victims, were engaged in outdoor and sports activities when injured or killed by lightning incidents.
\end{abstract}

Keyword: Injuries; Lightning casualties; Lightning fatalities; Lightning flash density 